\title{
ACTITUD DEL PROFESORADO DE EDUCACIÓN FÍSICA FRENTE AL APRENDIZAJE COOPERATIVO
}

\author{
PHYSICAL EDUCATION TEACHERS' ATTITUDE TOWARD COOPERATIVE \\ LEARNING
}

\author{
ATITUDE DOS PROFESSORES DE EDUCAÇÃO FÍSICA PERANTE A \\ APRENDIZAGEM COOPERATIVA
}

\author{
María Fernández Rivas ${ }^{*}$ María Espada Mateos ${ }^{*}$
}

\begin{abstract}
Palabras clave Educación Física.

Conducta cooperativa. Distribución por edad.

Actitud.

Resumen: El trabajo cooperativo tiene numerosos beneficios sobre los alumnos. En esta investigación se pretende conocer cuál es la actitud de los profesores de Educación Física ante el trabajo cooperativo y sus diferentes beneficios en función de la titulación y la edad, a partir de una muestra de 455 profesores de Educación Física, utilizando un análisis inferencial con diferentes tests (Welch y ANOVA). Algunos de los datos muestran diferencias significativas en función de la titulación, donde son los profesores licenciados o graduados en Ciencias de la Actividad Física y el Deporte y los diplomados o graduados en Educación Física los que consideran que el trabajo cooperativo mejora las relaciones interpersonales entre los alumnos $(p=.000)$, y que mejora la comunicación $(\mathrm{p}=.003)$. Además, respecto a la edad, los docentes más jóvenes creen que el trabajo cooperativo mejora las habilidades de interacción social $(p=.000)$.
\end{abstract}

Keywords Physical Education. Cooperative behavior. Age distribution. Attitude.

Palavras-chave Educação Física. Comportamento cooperativo. Distribuição por idade. Atitude

Abstract: Cooperative work has several advantages for students. In this research, we expect to find out Physical Education teachers' attitude towards cooperative learning style and its benefits according to teachers' educational level and age through a sample of 455 participants, using inferential analysis (Welch and ANOVA). Some data shows significant differences based on educational level, since teachers holding degrees in Sports and Physical Activity Science and Physical Education think that cooperative learning improves personal relationship ( $p=.000)$ and communication among students ( $p=.003)$. In addition, regarding age, younger teachers see cooperative learning as improving social interaction skills $(p=.000)$.

Resumo: 0 trabalho cooperativo tem numerosos benefícios sobre os alunos. Nesta investigação pretende-se conhecer qual é a atitude dos professores de Educação Física em relação ao trabalho cooperativo e seus diferentes benefícios em função da titulação e idade a partir de uma mostra de 455 professores de Educação Física, utilizando uma análise inferencial com diferentes testes (Welch y ANOVA). Alguns dados mostram diferenças significativas em função da titulação, sendo os professores licenciados ou graduados em Ciências da Atividade Física e Esporte e os diplomados ou graduados em Educação Física os que consideram que o trabalho cooperativo melhora as relações interpessoais entre os alunos ( $p=000)$ e a comunicação $(p=.003)$. Ademais, em relação à idade, os docentes mais jovens creem que o trabalho cooperativo melhora as habilidades de interação social $(p=.000)$.
*Universidad Camilo José Cela. Madrid, Espanha.

E-mail: maria_moremad@hotmail.com Recebido em: 14-01-2016 Aprovado em: 07-04-2016 (c) (1) (8) Licence 


\section{INTRODUCCIÓN}

Debido a la implantación de competencias en el nuevo sistema educativo, el trabajo cooperativo presenta un papel importante en el proceso de enseñanza-aprendizaje, ya que algunas de estas competencias consisten en el trabajo en equipo y habilidades sociales. Por ejemplo, la competencia aprender a aprender se puede manifestar de forma individual o en grupo intentando que los alumnos se conciencien de lo que hacen para aprender y busquen diferentes alternativas, son obtenidas más fácilmente a través del trabajo cooperativo. Para ello, es necesario saber cómo plantear los objetivos y cómo llevar a cabo este trabajo en el aula (BOE, 2015; SERRANO; PONS, 2014; TORREGO; NEGRO, 2012).

En la misma línea, Zabala (2003) considera la necesidad de mejorar las competencias profesionales de los estudiantes universitarios de cara a su inserción laboral, clasificándolas en competencias referidas a comportamientos profesionales y sociales, a las actitudes, a las capacidades creativas y a las competencias éticas.

Así, Johnson, Johnson y Holubec (1999) hacen referencia a la cooperación como una estrategia que permite a los alumnos trabajar juntos teniendo que lograr objetivos comunes. De esta forma, se observan numerosos beneficios como la inclusión, relaciones sociales, etc. (LAVEGA; PLANAS; RUIZ, 2014).

Concretamente, las ventajas de estructurar la educación en el aula a partir de actividades cooperativas son muchas (SANTOS-REGO; LORENZO-MOLEDO; PRIEGUE CAAMAÑO, 2009):

\footnotetext{
- Ayuda a los alumnos a asumir responsabilidades a la vez que se genera entre ellos la ayuda mutua. Esto es gracias a la motivación que se presenta en los alumnos para conseguir, juntos, un objetivo común.

- Da lugar a una educación basada en la democracia, un tema incluido en muchos de los sistemas educativos.

- Favorece la empatía hacia la diversidad y heterogeneidad, lo cual es importante dada la multiculturalidad que se presenta en la mayoría de las aulas.
}

Además, son muchas las investigaciones que afirman que el trabajo cooperativo aporta numerosos beneficios en Educación Física. Así, en las investigaciones de Dyson (2001) y Prieto y Nistal (2009), se afirma que el trabajo cooperativo permite a los alumnos mejorar de forma significativa el nivel físico en el contenido de condición física cuando lo aplican, y de manera general en la práctica motriz. También, Fernández Río (2003) y Prieto y Nistal (2009) afirman que la motivación del alumno por la asignatura aumenta significativamente al aplicar este trabajo, además de mejorar la autoestima. Por último, también se aprecia una mejora de la interacción social entre los alumnos, y por lo tanto una mejora a nivel afectivo-social (PRIETO; NISTAL, 2009).

Por otro lado, es importante conocer las diferentes situaciones de aprendizaje y sus características (SIMONI; SANTILLANA; YÁÑEZ, 2013, p.24): 
Cuadro 1- Elementos de las situaciones de aprendizaje

\begin{tabular}{|l|l|l|l|}
\hline Elementos & Estructura competitiva & Estructura individualista & Estructura cooperativa \\
\hline Trabajo individual & $\begin{array}{l}\text { Trabajo individual para } \\
\text { competir. }\end{array}$ & Trabajo individual. & $\begin{array}{l}\text { Trabajo individual para } \\
\text { cooperar. }\end{array}$ \\
\hline Tipo de ayuda & $\begin{array}{l}\text { No se tolera la ayuda } \\
\text { mutua. }\end{array}$ & Ayuda mutua tolerada. & $\begin{array}{l}\text { Se fomenta la ayuda } \\
\text { mutua. }\end{array}$ \\
\hline Tipo de independencia & Interdependencia negativa. & No hay interdependencia. & Interdependencia positiva. \\
\hline Tipo de nota & Nota normativa. & $\begin{array}{l}\text { Nota de progreso indivi- } \\
\text { dual. }\end{array}$ & $\begin{array}{l}\text { Nota de equipo (progre- } \\
\text { so). }\end{array}$ \\
\hline Incidencia del profesor & Enseña y resuelve dudas. & No llega a todos. & $\begin{array}{l}\text { Los alumnos también se } \\
\text { enseñan entre ellos. }\end{array}$ \\
\hline Autonomía & Poca autonomía. & Poca autonomía. & Más autonomía. \\
\hline Tipo de motivación & Motivación extrínseca. & Depende de cada alumno. & Motivación intrínseca. \\
\hline
\end{tabular}

Fuente: SIMONI et al., 2013, p.24.

En esta línea, la razón de la importancia dada al trabajo cooperativo se da en función de dos puntos de vista justificados por diferentes autores. La primera razón es desde un punto de vista escolar, ya que es necesario tener en cuenta las dimensiones psicosociológicas del aula, además de tener en cuenta que las relaciones alumno-alumno son fundamentales. La segunda, es desde un punto de vista social, ya que hoy en día son imprescindibles las relaciones interpersonales en la vida diaria. Así, algunas investigaciones afirman que los profesores de Educación Física dan mayor importancia a la enseñanza de valores, lo cual pertenece a un ámbito multidisciplinar, que a la consecución de los objetivos específicos de la Educación Física, lo que da lugar a una escasez de conocimientos sobre la materia no llegando a cumplir todos los objetivos del curriculum oficial. En este caso, el uso del trabajo cooperativo sería fundamental, ya que se trabajan tanto las enseñanzas en valores como las propias de la asignatura, siendo ésta una solución a dicha situación (HERNÁNDEZ-ÁLVAREZ et al., 2010; HERNÁNDEZ- ÁLVAREZ et al., 2006; JOHNSON; JOHNSON, 1990; OVEJERO, 1990)

Por esto, se plantea el problema de la formación inicial y permanente del profesorado acerca del aprendizaje cooperativo, ya que es necesario conocer cómo organizar las tareas en el aula, además de saber cómo adaptar los métodos a una situación específica de enseñanza y aprendizaje (alumnos con necesidades educativas especiales, estilos de enseñanza del profesor, entorno educativo, etc.) (SERRANO et al., 2008).

De esta forma, Fraile (2004) habla de las estrategias para incluir en la formación del profesorado de Educación Física tanto las horas dedicadas a la docencia como las dedicadas a organizar y supervisar el trabajo realizado por los alumnos en el aula, buscando la autonomía y democracia de éstos en el aula.

Así, los efectos del aprendizaje cooperativo en los alumnos, son diferentes en función de las perspectivas teóricas, las cuales son (SLEVIN, 2014):

- La perspectiva motivacional, en la que se considera la motivación una de las partes principales en el proceso de enseñanza-aprendizaje; por esta razón, se intenta incentivar el trabajo cooperativo en los alumnos permitiendo crear un ambiente en el que el alumno buscará conseguir sus objetivos y los del grupo.

- La perspectiva de cohesión social, en la cual se pretende conseguir la cohesión grupal a través del aprendizaje cooperativo, buscando la interacción entre los alumnos para la consecución de sus objetivos, ayudándose los unos a los otros para conseguir el objetivo final. 
- Desde la perspectiva evolutiva, se pretende que los alumnos adquieran conceptos que les permitan evolucionar y aumentar su nivel a la hora de resolver problemas.

- Y la perspectiva de desarrollo cognitiva, donde lo que se busca es que el alumno adquiera conocimientos nuevos y tenga la capacidad de recordarlos posteriormente para relacionarlos con otros contenidos.

\section{OBJETIVOS}

- Analizar la actitud de los profesores de Educación Física ante el aprendizaje cooperativo en función de la titulación, mostrando las diferencias existentes entre los grupos.

- Describir la actitud de los profesores de Educación Física ante el aprendizaje cooperativo en función de la edad, mostrando las diferencias existentes entre los grupos.

\section{MATERIAL Y MÉTODOS}

Se lleva a cabo una investigación cuantitativa, siendo descriptiva y no experimental (ANGUERA, 1992; CEA D’ANCONA, 2001).

\section{MUESTRA}

Para llevar a cabo la investigación, se ha utilizado una muestra de 455 profesores de la etapa de primaria y secundaria. La primera hace referencia a la primera etapa de educación obligatoria, la cual abarca desde los 6 a los 12 años y está dividida en 6 cursos. Y la segunda, la etapa de secundaria, está compuesta por seis cursos, de los cuales, los cuatro primeros son obligatorios, lo que se denomina Educación Secundaria Obligatoria (ESO) abarcando desde los 13 a los 16 años, y los dos siguientes cursos que son opcionales (Bachillerato) de los 17 a los 18 años. Los profesores de la muestra, además, pertenecen a diferentes titulaciones y características sociodemográficas. Así, el 47,7\% de la muestra son diplomados o graduados en magisterio de Educación Física, el 21,1\% son licenciados o graduados en Ciencias de Actividad Física y Deporte y el 31,2\% de los profesores tienen ambas titulaciones. Por otro lado, respecto a la edad, el 17,4\% de la muestra se encuentran en el rango de edad de menos de 30 años, el $51,4 \%$ pertenece al rango de entre 31 y 40 años, un 19,8\% de los profesores tienen entre 41 y 50 años y el $11,4 \%$ de la muestra pertenece a profesores con una edad de 51 años o más. Por último, el $70,8 \%$ de la muestra son hombres, mientras que el $29,2 \%$ son mujeres.

Para estimar el universo general del estudio hemos usado listados indirectos, que posibiliten la utilización de unidades de la muestra, debido a la inexistencia de un censo específico de profesores de Educación Física que trabajan en los centros educativos de la Comunidad de Madrid. Por ello, se ha utilizado un listado de centros educativos de primaria y secundaria de la Comunidad de Madrid (COMUNIDAD DE MADRID, 2014) y otro de los municipios y población de dicha comunidad (INSTITUTO DE ESTADÍSTICA DE LA COMUNIDAD DE MADRID, 2013). Así, a partir de estos listados se suma un total de 1.659 centros educativos con estas características.

Para determinar el tamaño de la muestra, se ha utilizado la fórmula para poblaciones finitas, explicada por diferentes autores (CEA D'ANCONA, 2004; SIERRA BRAVO, 2001), donde 
respecto a la varianza poblacional se ha recurrido al supuesto considerado más desfavorable, siendo así "P" y "Q" iguales, teniendo el valor del 50\% cada uno de ellos, con un nivel de confianza de entre -2 sigmas y +2 sigmas, siendo valores de una distribución normal y estando situada la probabilidad en el $95,5 \%$, con un margen de error de $\pm 4,75 \%$ para la muestra establecida.

Una vez que se ha aplicado la fórmula, el tamaño de la muestra obtenido ha sido el de 455 unidades de la población.

Se ha llevado a cabo un diseño muestral probabilístico por conglomerados y estratificado, seleccionando primero los municipios, luego los centros y por último los profesores a encuestar en cada centro educativo, todo ello de forma aleatoria (RODRÍGUEZ OSUNA, 2002).

\section{INSTRUMENTO}

El instrumento utilizado para la recogida de los datos es el cuestionario a través de la entrevista, lo que se denomina por algunos autores como entrevista estandarizada a través de cuestionario (HEINEMANN, 2003). Otros autores lo denominan, entrevista estructurada (LUSSIER; KIMBALL, 2008; SIERRA BRAVO, 2001).

En este caso se trata de un cuestionario sobre el aprendizaje cooperativo, llamado cuestionario-escala de la actitud del profesorado frente a la innovación educativa mediante técnicas de trabajo cooperativo (CAPIC), el cual fue aplicado en el estudio de Traver y García (2007), teniendo un coeficiente de fiabilidad alfa de Cronbach siendo .9381, es decir, una media de fiabilidad bastante alta, además de tener unos índices de homogeneidad de (ri(t-i)) $>0.5$, lo que aumenta la consistencia del cuestionario.

\section{PROCEDIMIENTO}

La recogida de datos tuvo lugar durante el curso académico 2014- 2015, a lo que se le denomina estudio transversal (SIERRA BRAVO, 2001), llevado a cabo por una única persona favoreciendo la eficacia de los datos.

Para el análisis de los datos, se ha realizado un análisis descriptivo e inferencial, a través de tablas de frecuencias y diferentes test (Welch y ANOVA) a través del programa estadístico informático para el análisis de datos SPSS®, Versión 20.

\section{RESULTADOS}

Lo primero que se observa son las diferencias de medias, pudiendo apreciar que la diferencia más alta se encuentra en el segundo ítem acerca de si el trabajo cooperativo permite desarrollar las habilidades de interacción social entre los alumnos, siendo la diferencia de medias $\mathrm{DM}=4.55$ y la desviación típica $\mathrm{DT}=0.73$.

Así, respecto a la variable de titulación, lo primero que se observa es la prueba de homogeneidad de Levene, la cual muestra diferencias significativas en numerosos ítems.

Tras esto, los ítem en los que se observó significación son sometidos a la prueba de Welch, la cual muestra que en todos ellos existen diferencias significativas $(p<.05)$, siendo éstos ítems 
afirmaciones acerca de que el trabajo cooperativo mejora las relaciones interpersonales (ítem 1), permite desarrollar habilidades de interacción (ítem 2), favorece la integración de alumnos con necesidades educativas especiales (ítem 3), favorece la ayuda mutua y colaboración (ítem 4), permite la aportación de nuevas ideas (ítem 6), favorece hábitos de convivencia (ítem 9), ayuda a conocer la diversidad del grupo (ítem 11) y permite valoraciones positivas individuales (ítem 16).

Para conocer con mayor exactitud dónde se encuentran estas diferencias se recurre a la prueba post-hoc Games-Howell (tabla 1). Así, se observa que tanto los profesores con la titulación de licenciados o graduados en CC. Actividad Física y Deporte (CAFYD) ( $p=.000$ ) y diplomados o graduados en Educación Física $(p=.034)$ tienen una actitud más positiva que los profesores con ambas titulaciones hacia el trabajo cooperativo ya que mejora las relaciones interpersonales entre los alumnos (ítem 1,) en mayor medida que los profesores con ambas titulaciones. También, los docentes diplomados o graduados en Educación Física tienen una actitud más positiva que los profesores con ambas titulaciones hacia el trabajo cooperativo ya que permite desarrollar habilidades de interacción social (ítem 2, $p=.002$ ), favorece la ayuda mutua y colaboración (ítem $4, p=.022$ ), enriquece al grupo a través de nuevas ideas (ítem 6 , $\mathrm{p}=.004$ ), mejora los hábitos de convivencia (ítem $9, \mathrm{p}=.015$ ) y ayuda a conocer la diversidad social del grupo (ítem 11, $p=.019$. Por último, los diplomados o graduados en Educación Física tienen una actitud más positiva hacia el trabajo cooperativo que los licenciados o graduados en CC. Actividad Física y Deporte ya que favorece la integración de alumnos con necesidades educativas especiales (ítem $3, p=.029$ ) y permite la valoración positiva individual (ítem 16, $\mathrm{p}=.016)$.

Tabla 1- Prueba post- hoc de Games- Howell respecto a la variable de titulación.

\begin{tabular}{|c|c|c|c|c|c|}
\hline Ítems & $\begin{array}{l}\text { (I) Titulación } \\
\text { inicial }\end{array}$ & (J) Titulación inicial & $\begin{array}{l}\text { Diferencia de } \\
\text { medias (I-J) }\end{array}$ & $\begin{array}{l}\text { Error } \\
\text { típico }\end{array}$ & Sig. \\
\hline \multirow{6}{*}{$\begin{array}{l}\text { 1. El trabajo } \\
\text { cooperativo } \\
\text { (TC) mejorará } \\
\text { las relaciones } \\
\text { interpersonales entre } \\
\text { ellos }\end{array}$} & \multirow{2}{*}{$\begin{array}{l}\text { Diplomado/Grado } \\
\text { Magisterio }\end{array}$} & Licenciado/ Grado CAFYD & 0,1033 & 0,07681 & ,372 \\
\hline & & Diplomado y Licenciado &, $34861^{*}$ & 0,08538 & 0 \\
\hline & \multirow{2}{*}{$\begin{array}{l}\text { Licenciado/ Grado } \\
\text { CAFYD }\end{array}$} & Diplomado/Grado Magisterio & $-0,1033$ & 0,07681 & ,372 \\
\hline & & Diplomado y Licenciado & ,24531* & 0,09786 & 034 \\
\hline & \multirow{2}{*}{$\begin{array}{l}\text { Diplomado y } \\
\text { Licenciado }\end{array}$} & Diplomado/Grado Magisterio &,$- 34861^{*}$ & 0,08538 & 0 \\
\hline & & Licenciado/ Grado CAFYD &,$- 24531^{*}$ & 0,09786 & 034 \\
\hline \multirow{6}{*}{$\begin{array}{l}\text { 2. El TC permitirá } \\
\text { desarrollar } \\
\text { habilidades de } \\
\text { interacción social } \\
\text { entre ellos }\end{array}$} & \multirow{2}{*}{$\begin{array}{l}\text { Diplomado/Grado } \\
\text { Magisterio }\end{array}$} & Licenciado/ Grado CAFYD & 0,09989 & 0,09019 &, 511 \\
\hline & & Diplomado y Licenciado & ,29957* & 0,08535 & 002 \\
\hline & \multirow{2}{*}{$\begin{array}{l}\text { Licenciado/ Grado } \\
\text { CAFYD }\end{array}$} & Diplomado/Grado Magisterio & $-0,09989$ & 0,09019 &, 511 \\
\hline & & Diplomado y Licenciado & 0,19968 & 0,10844 & ,159 \\
\hline & \multirow{2}{*}{$\begin{array}{l}\text { Diplomado y } \\
\text { Licenciado }\end{array}$} & Diplomado/Grado Magisterio &,$- 29957^{\star}$ & 0,08535 &, 002 \\
\hline & & Licenciado/ Grado CAFYD & $-0,19968$ & 0,10844 & ,159 \\
\hline \multirow{6}{*}{$\begin{array}{l}\text { 3. El TC favorecerá } \\
\text { la integración } \\
\text { de los alumnos } \\
\text { con necesidades } \\
\text { especiales }\end{array}$} & \multirow{2}{*}{$\begin{array}{l}\text { Diplomado/Grado } \\
\text { Magisterio }\end{array}$} & Licenciado/ Grado CAFYD &, $27813^{*}$ & 0,10798 &, 029 \\
\hline & & Diplomado y Licenciado & 0,10002 & 0,09111 &, 516 \\
\hline & \multirow{2}{*}{$\begin{array}{l}\text { Licenciado/ Grado } \\
\text { CAFYD }\end{array}$} & Diplomado/Grado Magisterio &,$- 27813^{*}$ & 0,10798 &, 029 \\
\hline & & Diplomado y Licenciado & $-0,17811$ & 0,1227 & ,317 \\
\hline & \multirow{2}{*}{$\begin{array}{l}\text { Diplomado y } \\
\text { Licenciado }\end{array}$} & Diplomado/Grado Magisterio & $-0,10002$ & 0,09111 &, 516 \\
\hline & & Licenciado/ Grado CAFYD & 0,17811 & 0,1227 & ,317 \\
\hline
\end{tabular}


Continuación da tabla $1 \ldots$

\begin{tabular}{|c|c|c|c|c|c|}
\hline \multirow{6}{*}{$\begin{array}{l}\text { 4. El TC favorecerá } \\
\text { la ayuda mutua y } \\
\text { la valoración de la } \\
\text { colaboración }\end{array}$} & \multirow{2}{*}{$\begin{array}{l}\text { Diplomado/Grado } \\
\text { Magisterio }\end{array}$} & Licenciado/ Grado CAFYD & 0,10868 & 0,10021 &, 525 \\
\hline & & Diplomado y Licenciado & ,25070* & 0,09396 & ,022 \\
\hline & \multirow{2}{*}{$\begin{array}{l}\text { Licenciado/ Grado } \\
\text { CAFYD }\end{array}$} & Diplomado/Grado Magisterio & $-0,10868$ & 0,10021 &, 525 \\
\hline & & Diplomado y Licenciado & 0,14202 & 0,12012 & ,465 \\
\hline & \multirow{2}{*}{$\begin{array}{l}\text { Diplomado y } \\
\text { Licenciado }\end{array}$} & Diplomado/Grado Magisterio &,$- 25070^{*}$ & 0,09396 & ,022 \\
\hline & & Licenciado/ Grado CAFYD & $-0,14202$ & 0,12012 & ,465 \\
\hline \multirow{6}{*}{$\begin{array}{l}\text { 6. El TC enriquecerá } \\
\text { al grupo a través } \\
\text { de la aportación de } \\
\text { ideas nuevas }\end{array}$} & \multirow{2}{*}{$\begin{array}{l}\text { Diplomado/Grado } \\
\text { Magisterio }\end{array}$} & Licenciado/ Grado CAFYD & 0,18721 & 0,1012 & 157 \\
\hline & & Diplomado y Licenciado & ,29461* & 0,09207 &, 004 \\
\hline & \multirow{2}{*}{$\begin{array}{l}\text { Licenciado/ Grado } \\
\text { CAFYD }\end{array}$} & Diplomado/Grado Magisterio & $-0,18721$ & 0,1012 & ,157 \\
\hline & & Diplomado y Licenciado & 0,10739 & 0,11889 & ,639 \\
\hline & \multirow{2}{*}{$\begin{array}{l}\text { Diplomado y } \\
\text { Licenciado }\end{array}$} & Diplomado/Grado Magisterio &,$- 29461^{*}$ & 0,09207 &, 004 \\
\hline & & Licenciado/ Grado CAFYD & $-0,10739$ & 0,11889 & ,639 \\
\hline \multirow{6}{*}{$\begin{array}{l}\text { 9. El TC favorecerá } \\
\text { la adquisición de } \\
\text { los hábitos de } \\
\text { convivencia }\end{array}$} & \multirow{2}{*}{$\begin{array}{l}\text { Diplomado/Grado } \\
\text { Magisterio }\end{array}$} & Licenciado/ Grado CAFYD & 0,12572 & 0,08808 & ,33 \\
\hline & & Diplomado y Licenciado & ,22827* & 0,08167 &, 015 \\
\hline & \multirow{2}{*}{$\begin{array}{l}\text { Licenciado/ Grado } \\
\text { CAFYD }\end{array}$} & Diplomado/Grado Magisterio & $-0,12572$ & 0,08808 & ,33 \\
\hline & & Diplomado y Licenciado & 0,10255 & 0,1057 &, 596 \\
\hline & \multirow{2}{*}{$\begin{array}{l}\text { Diplomado y } \\
\text { Licenciado }\end{array}$} & Diplomado/Grado Magisterio &,$- 22827^{*}$ & 0,08167 &, 015 \\
\hline & & Licenciado/ Grado CAFYD & $-0,10255$ & 0,1057 &, 596 \\
\hline \multirow{6}{*}{$\begin{array}{l}\text { 11. EI TC les } \\
\text { ayudará a conocer la } \\
\text { diversidad social del } \\
\text { propio grupo }\end{array}$} & \multirow{2}{*}{$\begin{array}{l}\text { Diplomado/Grado } \\
\text { Magisterio }\end{array}$} & Licenciado/ Grado CAFYD & 0,09164 & 0,09622 & ,608 \\
\hline & & Diplomado y Licenciado & ,26608* & 0,09798 &, 019 \\
\hline & \multirow{2}{*}{$\begin{array}{l}\text { Licenciado/ Grado } \\
\text { CAFYD }\end{array}$} & Diplomado/Grado Magisterio & $-0,09164$ & 0,09622 & 608 \\
\hline & & Diplomado y Licenciado & 0,17444 & 0,12012 & ,316 \\
\hline & \multirow{2}{*}{$\begin{array}{l}\text { Diplomado y } \\
\text { Licenciado }\end{array}$} & Diplomado/Grado Magisterio &,$- 26608^{*}$ & 0,09798 &, 019 \\
\hline & & Licenciado/ Grado CAFYD & $-0,17444$ & 0,12012 & ,316 \\
\hline \multirow{6}{*}{$\begin{array}{l}\text { 16. EI TC permitirá } \\
\text { la valoración positiva } \\
\text { las valoraciones } \\
\text { individuales }\end{array}$} & \multirow{2}{*}{$\begin{array}{l}\text { Diplomado/Grado } \\
\text { Magisterio }\end{array}$} & Licenciado/ Grado CAFYD & ,24890* & 0,1025 &, 043 \\
\hline & & Diplomado y Licenciado & 0,13637 & 0,0789 & ,197 \\
\hline & \multirow{2}{*}{$\begin{array}{l}\text { Licenciado/ Grado } \\
\text { CAFYD }\end{array}$} & Diplomado/Grado Magisterio &,$- 24890^{*}$ & 0,1025 &, 043 \\
\hline & & Diplomado y Licenciado & $-0,11253$ & 0,11275 &, 579 \\
\hline & \multirow{2}{*}{$\begin{array}{l}\text { Diplomado y } \\
\text { Licenciado }\end{array}$} & Diplomado/Grado Magisterio & $-0,13637$ & 0,0789 &, 197 \\
\hline & & Licenciado/ Grado CAFYD & 0,11253 & 0,11275 &, 579 \\
\hline
\end{tabular}

Por otro lado, respecto a los ítems en los cuales no se obtuvieron diferencias significativas a través de la prueba de homogeneidad de Levene, se realiza el test ANOVA, el cual muestra que existe significación $(p<.05)$ acerca de si el trabajo cooperativo hace tomar conciencia de que todos aprendan de todos (ítem 12), mejora la responsabilidad del alumno (ítem 14) y favorece la comunicación entre ellos (ítem 18).

Para clarificar dichas diferencias se realiza la prueba post-hoc Tukey (tabla 2), observando que éstas se encuentran entre diplomados o graduados en Educación Física y docentes con ambas titulaciones, siendo los diplomados o graduados en magisterio los que tienen una actitud m.as positiva hacia el trabajo cooperativo que los profesores con dos titulaciones ya que éste hace tomar conciencia de que todos aprendan de todos $(p=.025)$ y ayuda a que el alumno se sienta más responsable $(p=.048)$. También, tanto los diplomados o graduados en magisterio $(p=.018)$ como los licenciados o graduados en CC. Actividad Física y Deporte $(p=.004)$ tienen una actitud más positiva hacia el trabajo 
cooperativo que los profesores con dos titulaciones debido a que favorece la comunicación entre los alumnos.

Tabla 2- Prueba post- hoc de Tukey respecto a la variable de titulación.

\begin{tabular}{|c|c|c|c|c|c|}
\hline Ítems & $\begin{array}{l}\text { (I) Titulación } \\
\text { inicial }\end{array}$ & (J) Titulación inicial & $\begin{array}{c}\text { Diferencia de } \\
\text { medias (I-J) }\end{array}$ & $\begin{array}{l}\text { Error } \\
\text { típico }\end{array}$ & Sig. \\
\hline \multirow{6}{*}{$\begin{array}{l}\text { 12. EI TC hará tomar } \\
\text { conciencia de que } \\
\text { todos aprenden de } \\
\text { todos }\end{array}$} & \multirow{2}{*}{$\begin{array}{l}\text { Diplomado/Grado } \\
\text { Magisterio }\end{array}$} & Licenciado/ Grado CAFYD & 0,11367 & 0,09302 & ,441 \\
\hline & & Diplomado y Licenciado &, $21461^{*}$ & 0,08191 & 025 \\
\hline & \multirow{2}{*}{$\begin{array}{l}\text { Licenciado/ Grado } \\
\text { CAFYD }\end{array}$} & Diplomado/Grado Magisterio & $-0,11367$ & 0,09302 & ,441 \\
\hline & & Diplomado y Licenciado & 0,10094 & 0,10027 &, 573 \\
\hline & \multirow{2}{*}{$\begin{array}{l}\text { Diplomado y } \\
\text { Licenciado }\end{array}$} & Diplomado/Grado Magisterio &,$- 21461^{*}$ & 0,08191 & 025 \\
\hline & & Licenciado/ Grado CAFYD & $-0,10094$ & 0,10027 &, 573 \\
\hline \multirow{6}{*}{$\begin{array}{l}\text { 14. EI TC ayudará a } \\
\text { hacer sentir al alum- } \\
\text { no más responsable } \\
\text { de las tareas }\end{array}$} & \multirow{2}{*}{$\begin{array}{l}\text { Diplomado/Grado } \\
\text { Magisterio }\end{array}$} & Licenciado/ Grado CAFYD & 0,25427 & 0,10983 & 055 \\
\hline & & Diplomado y Licenciado & ,22860* & 0,09671 & 048 \\
\hline & \multirow{2}{*}{$\begin{array}{l}\text { Licenciado/ Grado } \\
\text { CAFYD }\end{array}$} & Diplomado/Grado Magisterio & $-0,25427$ & 0,10983 & 055 \\
\hline & & Diplomado y Licenciado & $-0,02567$ & 0,11839 & ,974 \\
\hline & \multirow{2}{*}{$\begin{array}{l}\text { Diplomado y } \\
\text { Licenciado }\end{array}$} & Diplomado/Grado Magisterio &,$- 22860^{*}$ & 0,09671 & 048 \\
\hline & & Licenciado/ Grado CAFYD & 0,02567 & 0,11839 & ,974 \\
\hline \multirow{6}{*}{$\begin{array}{l}\text { 18. EI TC favorecerá } \\
\text { la comunicación } \\
\text { entre ellos }\end{array}$} & \multirow{2}{*}{$\begin{array}{l}\text { Diplomado/Grado } \\
\text { Magisterio }\end{array}$} & Licenciado/ Grado CAFYD & $-0,116$ & 0,114 &, 565 \\
\hline & & Diplomado y Licenciado &, $275^{*}$ & 0,1 & 018 \\
\hline & \multirow{2}{*}{$\begin{array}{l}\text { Licenciado/ Grado } \\
\text { CAFYD }\end{array}$} & Diplomado/Grado Magisterio & 0,116 & 0,114 &, 565 \\
\hline & & Diplomado y Licenciado &, $391^{*}$ & 0,123 &, 004 \\
\hline & \multirow{2}{*}{$\begin{array}{l}\text { Diplomado y } \\
\text { Licenciado }\end{array}$} & Diplomado/Grado Magisterio &,$- 275^{*}$ & 0,1 &, 018 \\
\hline & & Licenciado/ Grado CAFYD &,$- 391^{*}$ & 0,123 &, 004 \\
\hline
\end{tabular}

De igual modo, respecto a la variable edad, lo primero que se realiza es la prueba de homogeneidad de Levene, donde se observan diferentes ítems con diferencias de varianzas $(p<.05)$.

Respecto a los ítems donde se obtuvieron estas diferencias, se recurre a la prueba Welch, la cual indica en qué ítems hay diferencias entre las variables. Así, estos ítems son los que afirman que el trabajo cooperativo permite la interacción social entre los alumnos (ítem $2 ; p=.000$ ), favorece la ayuda mutua y cooperación (ítem $4 ; p=.014$ ), permite a los alumnos comprender mejor a los demás (ítem 8; $p=.014$ ), favorece los hábitos de convivencia (ítem 9 ; $\mathrm{p}=.000$ ) y favorece la socialización de los alumnos (ítem 10; $\mathrm{p}=.019$ ).

Se lleva a cabo la prueba post-hoc Games- Howell (tabla 3), para conocer en que categorías se dan estas diferencias dentro de los ítems citados. Así, se observa que los docentes con la edad comprendida entre 31 y 40 años tienen una actitud más positiva hacia el trabajo cooperativo en comparación con los profesores de más edad (de 41 a 50 y más de 51 años) ya que permite desarrollar habilidades de interacción social y favorece la adquisición de hábitos de convivencia frente a los docentes de más edad. También, los profesores de edades comprendidas entre 31 y 40 años tienen una actitud más positiva hacia el trabajo cooperativo frente a los profesores con una edad comprendida entre 41 y 50 años ya que favorece la socialización de los alumnos (ítem 10). Por último, los docentes menores de 30 años presentan una actitud más positiva hacia el trabajo cooperativo que los profesores mayores de 51 años ya que éste permite comprender mejor a los demás (ítem 8). 
Tabla 3 - Prueba post- hoc Games- Howell respecto a la variable edad.

\begin{tabular}{|c|c|c|c|c|c|}
\hline Ítems & (I) Edad & (J) Edad & $\begin{array}{l}\text { Diferencia de } \\
\text { medias (I-J) }\end{array}$ & $\begin{array}{l}\text { Error } \\
\text { típico }\end{array}$ & Sig. \\
\hline \multirow{12}{*}{$\begin{array}{l}\text { 2. EI TC permitirá desarrollar habilida- } \\
\text { des de interacción social entre ellos }\end{array}$} & \multirow{3}{*}{30 o menos } & $31-40$ & $-0,09753$ & 0,09082 & ,706 \\
\hline & & $41-50$ & 0,25204 & 0,12153 & ,166 \\
\hline & & 51 o más & 0,43452 & 0,17995 & ,083 \\
\hline & \multirow{3}{*}{$31-40$} & 30 o menos & 0,09753 & 0,09082 & ,706 \\
\hline & & $41-50$ & ,34957* & 0,09547 & ,002 \\
\hline & & 51 o más &, $53205^{\star}$ & 0,16348 & ,01 \\
\hline & \multirow{3}{*}{$41-50$} & 30 o menos & $-0,25204$ & 0,12153 & , 166 \\
\hline & & $31-40$ &,$- 34957^{*}$ & 0,09547 & ,002 \\
\hline & & 51 o más & 0,18248 & 0,18234 & ,749 \\
\hline & \multirow{3}{*}{51 o más } & 30 o menos & $-0,43452$ & 0,17995 & ,083 \\
\hline & & $31-40$ &,$- 53205^{\star}$ & 0,16348 & ,01 \\
\hline & & $41-50$ & $-0,18248$ & 0,18234 &, 749 \\
\hline \multirow{12}{*}{$\begin{array}{l}\text { 4. EI TC favorecerá la ayuda mutua y } \\
\text { la valoración de la colaboración }\end{array}$} & \multirow{3}{*}{30 o menos } & $31-40$ & $-0,10381$ & 0,1132 & ,796 \\
\hline & & $41-50$ & 0,18594 & 0,14721 &, 588 \\
\hline & & 51 o más & 0,19961 & 0,14961 &, 543 \\
\hline & \multirow{3}{*}{$31-40$} & 30 o menos & 0,10381 & 0,1132 & ,796 \\
\hline & & $41-50$ & 0,28974 & 0,11519 & ,062 \\
\hline & & 51 o más & 0,30342 & 0,11824 & ,059 \\
\hline & \multirow{3}{*}{$41-50$} & 30 o menos & $-0,18594$ & 0,14721 &, 588 \\
\hline & & $31-40$ & $-0,28974$ & 0,11519 & ,062 \\
\hline & & 51 o más & 0,01368 & 0,15113 & 1 \\
\hline & \multirow{3}{*}{51 o más } & 30 o menos & $-0,19961$ & 0,14961 &, 543 \\
\hline & & $31-40$ & $-0,30342$ & 0,11824 & ,059 \\
\hline & & $41-50$ & $-0,01368$ & 0,15113 & 1 \\
\hline \multirow{12}{*}{$\begin{array}{l}\text { 8. EI TC permitirá que los alumnos } \\
\text { se pongan en el lugar de los demás y } \\
\text { comprendan mejor }\end{array}$} & \multirow{3}{*}{30 o menos } & $31-40$ & 0,11349 & 0,10034 & ,671 \\
\hline & & $41-50$ & 0,28101 & 0,13213 & 149 \\
\hline & & 51 o más &, $55794^{*}$ & 0,18964 & ,022 \\
\hline & \multirow{3}{*}{$31-40$} & 30 o menos & $-0,11349$ & 0,10034 & ,671 \\
\hline & & $41-50$ & 0,16752 & 0,11263 & ,448 \\
\hline & & 51 o más & 0,44444 & 0,17661 & ,067 \\
\hline & \multirow{3}{*}{$41-50$} & 30 o menos & $-0,28101$ & 0,13213 & 149 \\
\hline & & $31-40$ & $-0,16752$ & 0,11263 & ,448 \\
\hline & & 51 o más & 0,27692 & 0,19642 & ,497 \\
\hline & \multirow{3}{*}{51 o más } & 30 o menos &,$- 55794^{*}$ & 0,18964 & ,022 \\
\hline & & $31-40$ & $-0,44444$ & 0,17661 & ,067 \\
\hline & & $41-50$ & $-0,27692$ & 0,19642 & ,497 \\
\hline
\end{tabular}


Continuación da tabla 3

9. El TC favorecerá la adquisición de los hábitos de convivencia

\begin{tabular}{|c|c|c|c|c|}
\hline & $31-40$ & $-0,02867$ & 0,08887 & ,988 \\
\hline \multirow[t]{3}{*}{30 o menos } & $41-50$ & 0,22518 & 0,11585 & ,214 \\
\hline & 51 o más & 0,37731 & 0,15897 & ,09 \\
\hline & 30 o menos & 0,02867 & 0,08887 & ,988 \\
\hline \multirow[t]{3}{*}{$31-40$} & $41-50$ & ,25385* & 0,09241 & ,034 \\
\hline & 51 o más & ,40598* & 0,1428 & ,03 \\
\hline & 30 o menos & $-0,22518$ & 0,11585 & ,214 \\
\hline \multirow[t]{3}{*}{$41-50$} & $31-40$ &,$- 25385^{*}$ & 0,09241 & ,034 \\
\hline & 51 o más & 0,15214 & 0,16098 & ,781 \\
\hline & 30 o menos & $-0,37731$ & 0,15897 & ,09 \\
\hline \multirow[t]{3}{*}{51 o más } & $31-40$ &,$- 40598^{*}$ & 0,1428 &, 03 \\
\hline & $41-50$ & $-0,15214$ & 0,16098 & ,781 \\
\hline & $31-40$ & $-0,02029$ & 0,095 & ,997 \\
\hline \multirow[t]{3}{*}{30 o menos } & $41-50$ & 0,27117 & 0,1284 & ,154 \\
\hline & 51 o más & 0,19766 & 0,13699 & ,476 \\
\hline & 30 o menos & 0,02029 & 0,095 & ,997 \\
\hline \multirow[t]{3}{*}{$31-40$} & $41-50$ & ,29145* & 0,1041 & 0,03 \\
\hline & 51 o más & 0,21795 & 0,11453 & ,237 \\
\hline & 30 o menos & $-0,27117$ & 0,1284 & ,154 \\
\hline \multirow[t]{3}{*}{$41-50$} & $31-40$ &,$- 29145^{\star}$ & 0,1041 & ,03 \\
\hline & 51 o más & $-0,0735$ & 0,14345 & ,956 \\
\hline & 30 o menos & $-0,19766$ & 0,13699 &, 476 \\
\hline \multirow[t]{3}{*}{51 o más } & $31-40$ & $-0,21795$ & 0,11453 & ,237 \\
\hline & $41-50$ & 0,0735 & 0,14345 & ,956 \\
\hline & $31-40$ & 0,00909 & 0,10799 & 1 \\
\hline \multirow[t]{3}{*}{30 o menos } & $41-50$ & 0,19114 & 0,13347 & ,481 \\
\hline & 51 o más & 0,40652 & 0,19677 & ,173 \\
\hline & 30 o menos & $-0,00909$ & 0,10799 & 1 \\
\hline \multirow[t]{3}{*}{$31-40$} & $41-50$ & 0,18205 & 0,10886 & ,342 \\
\hline & 51 o más & 0,39744 & 0,18098 & ,136 \\
\hline & 30 o menos & $-0,19114$ & 0,13347 & ,481 \\
\hline \multirow[t]{3}{*}{$41-50$} & $31-40$ & $-0,18205$ & 0,10886 & ,342 \\
\hline & 51 o más & 0,21538 & 0,19725 & ,695 \\
\hline & 30 o menos & $-0,40652$ & 0,19677 &, 173 \\
\hline \multirow[t]{2}{*}{51 o más } & $31-40$ & $-0,39744$ & 0,18098 & ,136 \\
\hline & $41-50$ & $-0,21538$ & 0,19725 & ,695 \\
\hline
\end{tabular}

10. El TC favorecerá y potenciará la socialización de los mismos

14. El TC ayudará a hacer sentir al alumno más responsable de las tareas

${ }^{*} \mathrm{TC}$ : trabajo cooperativo.

Fuente: Elaboración propia.

Los ítems en los que no existen diferencias de varianzas en la prueba de homogeneidad de Levene, son sometidos al estadístico de correlación ANOVA sin mostrar ningún dato significativo en los resultados, ya que en todos los casos p>.05. 


\section{DISCUSIÓN}

Respecto a la titulación, cabe destacar que los docentes con la licenciatura o grado en Ciencias de Actividad Física y Deporte y los diplomados o graduados en Educación Física tienen una actitud positiva hacia el trabajo cooperativo ya que mejora las relaciones interpersonales entre los alumnos y la comunicación entre ellos en mayor medida que los profesores con ambas titulaciones. También, los profesores que son diplomados o graduados en Educación Física muestran una actitud más positiva hacia el trabajo cooperativo debido a que favorece las relaciones sociales así como la colaboración entre los alumnos en comparación con los otros grupos. Pegalajar y Colmenero (2013) y De la Torre (2000) es sus investigaciones afirman la actitud positiva por parte de los sujetos encuestados, la cual varía en función de la formación inicial, a la hora de llevar a cabo esta metodología ya que se potencian numerosas capacidades, como la discusión de grupo y el trabajo en equipo, situando al trabajo cooperativo dentro de una metodología creativa. Así, existen numerosas investigaciones que afirman que el aprendizaje cooperativo mejora las relaciones sociales entre los alumnos y el apoyo social (BARBA, 2010; DUNN; WILSON, 1991; DYSON, 2001; GOUDAS; MAGOTSIOU, 2009; JOYCE, WEIL, 2002; POLVI; TELAMA, 2000; VELÁZQUEZ, 2004). Además, el trabajo cooperativo permite que los alumnos asuman responsabilidades a la vez que se genera la ayuda mutua entre ellos, permitiendo una mejora de la interacción social, lo que hace que los profesores acepten el aprendizaje cooperativo como forma de trabajo en el aula de Educación Física (PRIETO; NISTAL, 2009; SANTOS-REGO; LORENZOMOLEDO; PRIEGUE CAAMAÑO, 2009).

En la misma línea, se observa que respecto a la edad existen diferentes actitudes en cuanto que el trabajo cooperativo mejora las habilidades de interacción social favoreciendo los hábitos de convivencia, siendo los docentes más jóvenes con edades comprendidas entre los 31 y 40 años los que muestran una actitud más positiva frente a los profesores mayores de 41 años, y para la mejora de la socialización que los profesores con edades entre 41 y 50. Además, los profesores que tienen 30 años o menos, tienen una actitud más positiva hacia el trabajo cooperativo ya que permite que los alumnos comprendan mejor a los demás y permite que éstos se pongan en el lugar del resto de alumnos. Pegalajar y Colmenero (2013) en su investigación afirman que los sujetos encuestados tienen una actitud más positiva frente al trabajo cooperativo según la edad, siendo los profesores que más conocimientos tienen sobre el tema los que tienen una actitud más positiva, lo que apoya los resultados obtenidos, ya que al ser ésta una herramienta metodológica innovadora, son los profesores más jóvenes los que tienen una actitud más positiva, todo ello gracias a los conocimientos obtenidos sobre el trabajo cooperativo a partir de su formación inicial. Por otro lado, existen numerosas investigaciones llevadas a cabo con el objetivo de conocer si el aprendizaje cooperativo mejora las relaciones sociales entre los alumnos, así como el apoyo social. Así, éstas se ven más favorecidas cuando las actividades en Educación Física se llevan a cabo a través del trabajo cooperativo (BARBA, 2010; DUNN; WILSON, 1991; DYSON, 2001; GOUDAS; MAGOTSIOU, 2009; OVEJERO, 1990; POLVI; TELAMA, 2000; SLAVIN; COOPER, 1999; VELÁZQUEZ, 2004). Por esto, los profesores de Educación Física presentan una actitud positiva hacia el trabajo cooperativo, ya que éste permite aportar numerosos beneficios a los alumnos, como la mejora de las capacidades afectivo-sociales, a la vez que permite a los alumnos conocer la heterogeneidad del grupo, siendo capaces de empatizar hacia la diversidad (PRIETO; NISTAL, 2009; SANTOS-REGO; 
LORENZO-MOLEDO; PRIEGUE CAAMAÑO, 2009; SANTOS-REGO; LORENZO-MOLEDANO, 2009)

Por esto, los profesores prefieren el uso del trabajo cooperativo a otro tipo de trabajo, como el competitivo, ya que los beneficios que aporta son mayores y permiten mejorar más habilidades tanto sociales como de rendimiento (GOUDAS Y MAGOTSIOU, 2009). Todos estos beneficios en las habilidades de los alumnos, son debidos a un aumento de la motivación producida por este tipo de trabajo, permitiendo que éstos se impliquen en mayor medida en las tareas, aumentando la atención, participación y cooperación entre ellos, valorando la Educación Física con una actitud más positiva (BARBA, 2010; DUNN; WILSON, 1991; DYSON, 2001; FERNÁNDEZ-RÍO, 2003; GOUDAS; MAGOTSIOU, 2009; JOYCE; WEIL, 2002; OVEJERO, 1990; POLVI; TELAMA, 2000; VELÁZQUEZ, 2004). Los profesores de Educación Física conocen las ventajas del trabajo cooperativo, siendo ésta la razón por la que presenta una actitud positiva hacia él. Gracias a este trabajo los alumnos aumentarán su motivación hacia la asignatura, además de su autoestima, y como consecuencia su rendimiento en los diferentes canales de desarrollo (FELDER; BRENT, 2001; FERNÁNDEZ RÍO, 2003; PEGALAJAR; COLMENERO, 2013; PRIETO; NISTAL, 2009; SANTOS-REGO; LORENZO-MOLEDO; PRIEGUE CAAMAÑO, 2009).

\section{CONCLUSIONES}

Los profesores con edades entre 31 y 40 años tienen una actitud más positiva hacia el trabajo cooperativo que los profesores que tienen entre 41 y 50 años ya que potencia la socialización. También, estos profesores tienen una actitud más positiva que los profesores mayores de 41 años ya que consideran que mejora los hábitos de convivencia e interacción social. Además, existe una actitud positiva hacia este trabajo ya que permite que los alumnos se pongan en el lugar de los demás, principalmente para profesores menores de 30 años frente a los más veteranos, mayores de 51 años.

Respecto a la titulación, son los profesores licenciados o graduados en Ciencias de Actividad Física y Deporte y diplomados o graduados en magisterio de Educación Física quienes tienen una actitud más positiva que los profesores con ambas titulaciones hacia el trabajo cooperativo ya que consideran que mejora la interacción social, la ayuda mutua y la colaboración entre los alumnos, permite aportar nuevas ideas y conocer la diversidad del aula, aprendiendo todos de todos y haciendo que se sientan más responsables. También, los profesores que son diplomados o graduados en magisterio de Educación Física tienen una actitud más positiva que los profesores licenciados o graduados en Ciencias de Actividad Física y Deporte hacia el trabajo cooperativo ya que favorece la integración y permite que los alumnos realicen valoraciones individuales positivas. Por último, tanto licenciados o graduados en Ciencias de Actividad Física y Deporte como diplomados o graduados en magisterio de Educación Física tienen una actitud más positiva hacia el trabajo cooperativo que los profesores con ambas titulaciones ya que éste permite mejorar las relaciones interpersonales entre los alumnos y la comunicación entre ellos.

De esta forma, se concluye de manera general que los profesores de Educación Física tienen una actitud positiva hacia el aprendizaje cooperativo ya que mejora las actitudes y capacidades de los alumnos, lo que permite un mejor funcionamiento de la clase para alcanzar los objetivos propuestos. 


\section{REFERENCIAS}

ANGUERA, María Teresa. Metodología de la Observación en las Ciencias Humanas. Madrid: Catedra, 1992.

BARBA, José Juan. Diferencias Entre Aprendizaje Cooperativo y la Asignación de Tareas. en la Escuela Rural: Comparación de dos Estudios de Caso en una Unidad Didáctica de Acrosport en Segundo Ciclo de Primaria. Retos, Murcia, n.18, p.14-15, 2010.

CEA D’ANCONA, María Ángeles. Metodología Cuantitativa: Estrategias y Técnicas de Investigación Social. Madrid: Síntesis, 2001.

COMUNIDAD DE MADRID. Guía de centros docentes de la Comunidad de Madrid, 2014. Disponible en: <www.madrid.org/centros docentes/guia/index.html>. Acceso en: 9 set. 2014.

DE LA TORRE, Saturnino. Tres ideas en acción. Innovación-Formación-Investigación. En: DE LA TORRE, Saturnino; BARRIOS, Oscar (coords.). Estrategias didácticas innovadoras: Recursos para la formación y el cambio. Barcelona: Octaedro, p.391-392, 2000.

DUNN, Steven; WILSON, Rolayne. Cooperative Learning in the Physical Education Classroom. Journal of Physical Education, Recreation and Dance, Reston, v.62, n.6, p. 22-28, 1991.

DYSON, Ben. Cooperative Learning in an Elementary Physical Education Program, Journal of Teaching in Physical Education, Champaign, n.20, p.264-281, 2001.

FELDER, Richard.; BRENT, Rebecca. Effective strategies for cooperative learning. Journal Cooperation \& Collaboration in college teaching, v.10, n.2, p.69-75, 2001.

FERNÁNDEZ- RÍO, Javier. El Aprendizaje Cooperativo en el Aula de Educación Física para la Integración en el Medio Social: Análisis Comparativo con otros Sistemas de Enseñanza y Aprendizaje. Valladolid: La Peonza, 2003.

FRAILE, Antonio. Un Cambio Democrático en las Aulas Universitarias: unas Experiencias en la Formación del Profesorado de Educación Física. Contextos Educativos: Revista de Educación, La Rioja, v.7, n.6, p.213-234,2004.

GOUDAS, Marios; MAGOTSIOU Evmorfia. The Effects of a Cooperative Physical Education Program on Students' Social Skills. Journal of Applied Sport Psychology, Indianopolisis, v.21, n.3, p.356364,2009 .

HEINEMANN, Klaus. Introducción a la Metodología de la Investigación Empírica en las Ciencias del Deporte. Barcelona: Paidotribo, 2003.

HERNÁNDEZ-ALVAREZ, Juan Luis et al. Percepción del Esfuerzo en Educación Física y su Relación con las Directrices sobre Actividad Física. Revista Internacional de Medicina y Ciencias de Actividad Física y Deporte, Madrid, v.10, n.40, p.609-619, 2010.

HERNÁNDEZ- ALVAREZ, Juan Luis et al. La Formación en Conceptos en Educación Física: ¿Qué Saben los Alumnos de Educación Primaria? Revista Internacional de Medicina y Ciencias de Actividad Física y Deporte, Madrid, v.6, n.24, p.173-187, 2006.

INSTITUTO DE ESTADÍSTICA DE LA COMUNIDAD DE MADRID. Demografía y población, 2013. Disponible en: <http://www.madrid.org/iestadis>. Acceso en: 9 set.. 2014.

JOHNSON, David; JOHNSON Roger. Cooperation and Competition: Theory and Research. Edina Minnesota: Interaction Book, 1990. 
JOHNSON, David; JOHNSON, Roger; HOLUBEC Edythe. El Aprendizaje Cooperativo en el Aula. Buenos Aires: Paidós, 1999.

JOYCE, Bruce; WEIL, Marsha. Modelos de Enseñanza. Barcelo: Gedisa, 2002.

LAVEGA, Pere.; PLANAS, Antonio; RUIZ, Pedro. Juegos Cooperativos e Inclusión en Educación Física. Revista Internacional de Medicina y Ciencias de Actividad Física y Deporte, Madrid, v.14, n.53, p.37-51, 2014.

LUSSIER, Robert; KIMBALL, David. Applied Sport Management Skills. Champaign: Human Kinetics, 2008.

OVEJERO, Anastasio. El Aprendizaje Cooperativo. Barcelona: PPU, 1990.

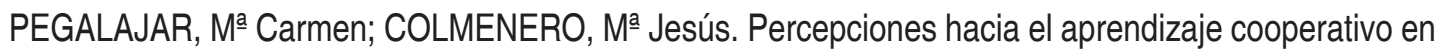
estudiantes del Grado de Maestro. Revista docente universitaria, v.11, n.3, p.343-362, 2013.

POLVI, Singa; TELAMA, Risto. The Use of Cooperative Learning as a Social Enhancer in Physical Education. Scandinavian Journal of Education Research, v.44, n.1, p.105-115, 2000.

PRIETO, José; NISTAL, Paloma. Influencia del aprendizaje cooperativo em educación física. Revista Iberoamericana de Educación, v.4, n.49, p.1-8, 2009.

RODRÍGUEZ OSUNA, Jacinto. El Análisis de la Realidad Social: Métodos y Técnicas de Investigación. 3. ed. Madrid: Alianza Editorial, 2002.

SANTOS-REGO, Miguel Ángel; LORENZO-MOLEDO, María del Mar. A educación para a cidadanía e os profesores: Visión e desafío. Vigo: Xerais, 2009.

SANTOS-REGO, Miguel Ángel; LORENZO-MOLEDO, María del Mar; PRIEGUE-CAAMAÑO, Diana. Aprendizaje cooperativo: práctica pedagógica para el desarrollo escolar y cultural. Revista Internacional de Investigación en Educación, Medelin, v.1, n.2, p. 289-303, 2009.

SERRANO, José Manuel et al. Evaluación del Programa de Formación de Profesores en Métodos de Aprendizaje Cooperativo Basadas en Análisis de Ecuaciones Estructurales. Revista Electrónica de Investigación Educativa, Ensenada, v.10, n.2, p.1-30 2008.

SERRANO, José Manuel; PONS Rosa María. Introduction: Cooperative Learning. Anales de Psicología, Murcia, v.30, n.3, p.781-784, 2014.

SIERRA BRAVO, Restituto. Técnicas de Investigación Social: Teoría y Ejercicios. Madrid: Thomson, 2001.

SIMONI, César; SANTILLANA,Hadi; YÁÑEZ, Arturo. La Inclusión y el Aprendizaje Cooperativo en la Sesión de Educación Física a través del Puzzle de Aronson. La Peonza, Santander, n.8, p.20-32, 2013.

SLAVIN, Robert; COOPER, Robert. Improving Intergroup Relations: Lessons Learned from Cooperative Learning Programs. Journal of Social Issues, México,DC, v.55, n.4, p.647-664. 1999.

SLEVIN, Robert. Cooperative Learning and Academic Achievement: Why Does Groupwork Work? Annals of Psychology, Murcia, v.30, n.3, p.785-791, 2014.

TORREGO, Juan Carlos.; NEGRO, Andrés. Trabajar y Aprender Contigo: Aprendizaje Cooperativo. Revista Electrónica de Investigación Educativa, Ensenada, v.14, n.2, p.289-296, 2012.

TRAVER, Joan Andrés; GARCÍA, Rafaela. Construcción de un Cuestionario-Escala sobre Actitud del Profesorado Frente a la Innovación Educativa Mediante Técnicas de Trabajo Cooperativo (CAPIC).

Revista Electrónica de Investigación Educativa, Ensenada, v.9, n.1, p.2-14, 2007. 
VELÁZQUEZ, Carlos. Las Actividades Físicas Cooperativas. México D.C.: Secretaría de Educación Pública, 2004.

ZABALA, Miguel Ángel. 2003. El Aprendizaje Experiencial Como Marco Teórico Para el prácticum. In: SYMPOSIUM INTERNACIONAL SOBRE EL PRÁCTICUM,7, 2003. Actas.... Pontevedra: Servicio de edición de la Universidad, 2003. p. 19-34. 
